\title{
Lithium production by thermohaline mixing in low-mass, low-metallicity asymptotic giant branch stars
}

\author{
Richard J. Stancliffe, George C. Angelou and John C. Lattanzio \\ Centre for Stellar and Planetary Astrophysics, Monash University, VIC 3800, Australia \\ email: Richard.Stancliffe@sci.monash.edu.au
}

\begin{abstract}
We examine the effects of thermohaline mixing on the composition of the envelopes of low-metallicity asymptotic giant branch (AGB) stars. We have evolved models of 1, 1.5 and $2 \mathrm{M}_{\odot}$ and of metallicity $Z=10^{-4}$ from the pre-main sequence to the end of the thermal pulsing asymptotic giant branch with thermohaline mixing applied throughout the simulations. We find that the small amount of ${ }^{3} \mathrm{He}$ that remains after the first giant branch is enough to drive thermohaline mixing on the AGB and that the mixing is most efficient in the early thermal pulses, with the efficiency dropping from pulse to pulse. We note a surprising increase in the ${ }^{7} \mathrm{Li}$ abundance, with $\log _{10} \epsilon\left({ }^{7} \mathrm{Li}\right)$ reaching values of over 2.5 in the $1.5 \mathrm{M}_{\odot}$ model. It is thus possible to get stars which are both $\mathrm{C}$ - and Li-rich at the same time. We compare our models to measurements of carbon and lithium in carbon-enhanced metal-poor stars which have not yet reached the giant branch. These models can simultaneously reproduced the observed $\mathrm{C}$ and $\mathrm{Li}$ abundances of carbon-enhanced metal-poor turn-off stars that are Li-rich.
\end{abstract}

Keywords. Stars: evolution, AGB and post-AGB, Population II, carbon

\section{Introduction}

It has long been known that models of asymptotic giant branch (AGB) stars that only include mixing in convective regions are incomplete. These canonical models cannot account for observations such as: the low ${ }^{12} \mathrm{C} /{ }^{13} \mathrm{C}$ ratios in low-mass AGB stars (Abia \& Isern 1997; Lebzelter et al. 2008), Li and C-rich stars in our Galaxy (Abia \& Isern 1997; Uttenthaler et al. 2007), isotopic ratios measured in pre-solar grains (e.g. Nollett et al. 2003, and references therein). It has therefore been suggested that material might circulate below the base of the convective envelope into regions where nuclear burning can happen. This process is often referred to as 'cool bottom processing'.

There have been detections of lithium in carbon-enhanced metal-poor (CEMP) stars (e.g. Thompson et al. 2008) and this is difficult to reconcile with standard models of AGB stars. Canonical AGB models produce Li via the Cameron-Fowler mechanism (Cameron \& Fowler 1971), which involves the production of beryllium deep in the hydrogen burning shell via the reaction ${ }^{4} \mathrm{He}\left({ }^{3} \mathrm{He}, \gamma\right){ }^{7} \mathrm{Be}$ and the immediate transport of this to cooler regions of the star where the ${ }^{7} \mathrm{Li}$ that forms (once the beryllium has undergone electron capture) is stable against proton captures. This takes place in the more massive stars which undergo hot bottom burning (HBB, where the base of the convective envelope lies in the top of the hydrogen-burning shell). Such stars would be rich in nitrogen, not carbon. These observations suggest that something is missing from the AGB models and an extra mixing mechanism must be at work.

Lithium is a particularly important element from the point of view of mixing processes in CEMP stars, especially those that are enriched in $s$-process elements. These CEMP-s stars are believed to have formed in binary systems where mass has been transferred 
from an AGB star which is no longer visible on to a companion that we now observe as carbon rich. Stancliffe et al. (2007) pointed out that material accreted on to a lowmass companion does not just remain at the surface of the companion star and that thermohaline mixing could efficiently mix this material deep into the stellar interior. However, detection of lithium in the CEMP binary system CS 22964-161 led Thompson et al. (2008) to suggest that the mixing efficiency could not be so high. Li is a fragile element and is easily destroyed at temperatures in excess of about $2.5 \times 10^{6} \mathrm{~K}$. Even a modest depth of mixing can lead to efficient Li-depletion (Stancliffe 2009) and hence the measurement of Li in CEMP stars could be a good test of the efficiency of thermohaline mixing. It is therefore crucial that we understand the origin of this element.

Despite the apparent need for extra mixing on both the giant branches, the physical nature of the mechanism (or mechanisms) has proved illusive. Recently, Eggleton et al. (2006) showed that the lowering of the mean molecular weight by the reaction ${ }^{3} \mathrm{He}\left({ }^{3} \mathrm{He}, 2 \mathrm{p}\right){ }^{4} \mathrm{He}$ could lead to mixing in red giants via the thermohaline instability. This can potentially explain the change in abundances seen in giants above the luminosity bump (Charbonnel \& Zahn 2007; Eggleton et al. 2008). In this work, we wish to examine what the consequences of thermohaline mixing are for low-mass, low-metallicity AGB stars.

\section{The stellar evolution code}

Calculations in this work have been carried out using the STARS stellar evolution code (see Stancliffe \& Eldridge 2009, for a detailed description of the code). Thermohaline mixing is included throughout all the evolutionary phases via the prescription of Kippenhahn, Ruschenplatt \& Thomas (1980), with the mixing coefficient being multiplied by a factor of 100 as suggested by the work of Charbonnel \& Zahn (2007). These authors find that with a factor of this magnitude they are able to reproduced the abundance trends observed towards the tip of the red giant branch. Stancliffe et al. (2009) also showed that a coefficient of this magnitude could reproduced the observed mixing trends in both low-mass metal-poor stars and carbon-enhanced metal-poor stars on the upper part of the first giant branch.

We evolve stars of $1,1.5$ and $2 \mathrm{M}_{\odot}$ from the pre-main sequence to the end of the thermally pulsing asymptotic giant branch (TP-AGB) using 999 mesh points. Reimers (1975) mass-loss prescription, with $\eta=0.4$, is used from the Main Sequence up to the TP-AGB; the Vassiliadis \& Wood (1993) mass-loss law is employed during the TP-AGB. A mixing length parameter of $\alpha=2.0$ is employed. The metallicity of each model is $Z=10^{-4}([\mathrm{Fe} / \mathrm{H}] \approx-2.3)$ and the initial abundances are assumed to be solar-scaled according to Anders \& Grevesse (1989), with the exception of ${ }^{7} \mathrm{Li}$, for which we adopt a value of $\mathrm{X}_{7} \mathrm{Li}=1.05 \times 10^{-9}$ which is equivalent to the Spite plateau value.

\section{Lithium production on the AGB}

While lithium is destroyed on the red giant branch, we find that it can be produced during the TP-AGB phase. Lithium production occurs in each of the models in the following way. First, the deepening of the convective envelope that occurs after every thermal pulse (referred to as third dredge-up) homogenises the envelope and flattens out the mean molecular weight above the burning shell (top left panel of Fig. 1). This is an essential precursor to thermohaline mixing. The reaction ${ }^{3} \mathrm{He}\left({ }^{3} \mathrm{He}, 2 \mathrm{p}\right){ }^{4} \mathrm{He}$ can only produce a small reduction of the mean molecular weight and its effects will only be apparent in a region of zero mean molecular weight gradient. 


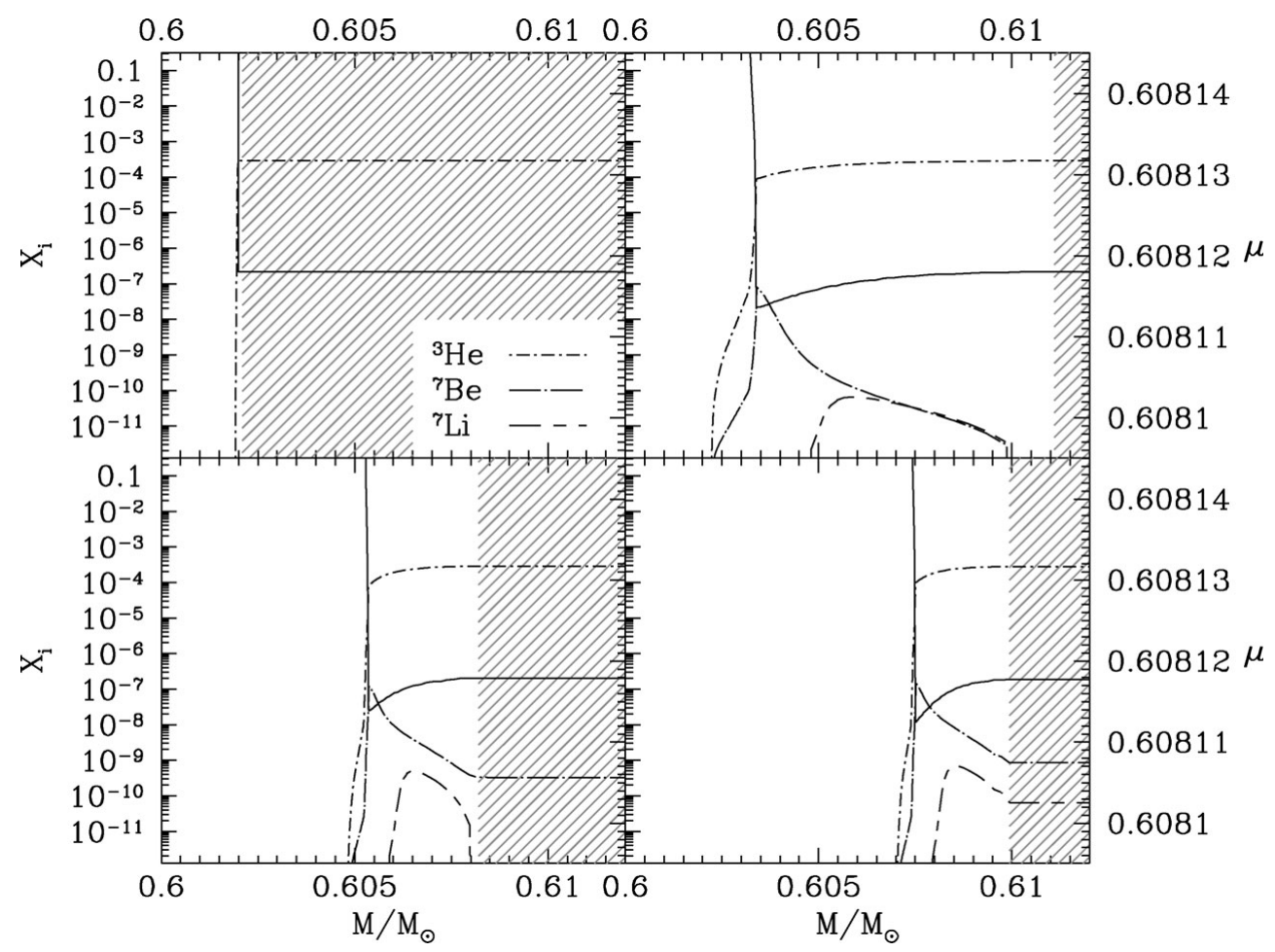

Figure 1. Abundance profiles of the $1.5 \mathrm{M}_{\odot}$ model after its second thermal pulse. In each panel, the abundances are: ${ }^{3} \mathrm{He}$ (dot-short dash line), ${ }^{7} \mathrm{Be}$ (dot-long dash line) and ${ }^{7} \mathrm{Li}$ (dashed line). The mean molecular weight, $\mu$, is displayed by a solid line. The grey shading indicates convective regions. Top left: Just after the end of third dredge-up. Top right: Retreat of the convective envelope. Thermohaline mixing leads to the formation of a pocket of beryllium and lithium. Bottom left:. The hydrogen shell moves outward and the envelope comes back in again. Bottom right Just before the beginning of the next thermal pulse.

After the convective envelope has reached its maximum depth, it begins to retreat and hydrogen burning re-ignites. At the top of the hydrogen burning shell, two important reactions are occurring. The ${ }^{3} \mathrm{He}\left({ }^{3} \mathrm{He}, 2 \mathrm{p}\right){ }^{4} \mathrm{He}$ lowers the mean molecular weight and thermohaline mixing starts to occur. In addition, the fusion of ${ }^{3} \mathrm{He}$ with ${ }^{4} \mathrm{He}$ produces ${ }^{7} \mathrm{Be}$. Thermohaline mixing transports this newly synthesised beryllium toward the convective envelope. En route, ${ }^{7} \mathrm{Be}$ undergoes electron capture to produce ${ }^{7} \mathrm{Li}$. While it remains deep in the star, this lithium is destroyed by proton captures. Thermohaline mixing is not efficient enough to transport the lithium to the envelope before it is destroyed. Pockets of ${ }^{7} \mathrm{Be}$ and ${ }^{7} \mathrm{Li}$ (top right panel of Fig. 1) are thus formed and their abundances depend on the rate at which fresh beryllium is transported up from hydrogen burning shell and the rate at which both species are being destroyed.

As the star settles into the interpulse phase, the convective envelope retreats inward again but not to the same extent as it did during third dredge-up. The envelope reaches into the lithium pocket that has been formed and a surface enrichment of lithium occurs. In addition the distance between the hydrogen burning shell and the convective envelope has now been reduced and thermohaline mixing is now able to transport lithium into the envelope before it is destroyed (bottom panels of Fig. 1). The lithium abundance thus continues to increase during the interpulse phase. 
We find that the $1.5 \mathrm{M}_{\odot}$ model obtains the greatest lithium enrichment of the three models we have run. By the time the star enters the superwind phase the surface lithium abundance has reached $\log _{10} \epsilon\left({ }^{7} \mathrm{Li}\right)=2.51$. The $2 \mathrm{M}_{\odot}$ model reaches a peak surface lithium enrichment of $\log _{10} \epsilon\left({ }^{7} \mathrm{Li}\right)=1.44$ after 7 thermal pulses but then suffers a slight decline as the base of the convective envelope becomes sufficiently deep for some lithium destruction to take place. At the onset of the superwind phase, the surface lithium abundance is $\log _{10} \epsilon\left({ }^{7} \mathrm{Li}\right)=1.38$. The $1 \mathrm{M}_{\odot}$ model is the least enriched by this mechanism and only reaches $\log _{10} \epsilon\left({ }^{7} \mathrm{Li}\right)=1.03$ by the end of the TP-AGB.

\section{Discussion}

The surprising outcome of these simulations is the high Li abundances that can be produced. It is usually supposed that only the higher mass AGB stars which undergo hot bottom burning are able to produce Li via the Cameron-Fowler mechanism (Cameron \& Fowler 1971). This work shows that it is possible that low-mass AGB stars could be producers of lithium- $\%$. We also note that the action of thermohaline mixing on the AGB is subtly different from its action of the RGB. On the RGB, it leads to a depletion of $\mathrm{Li}$ with the star leaving the RGB with virtually no lithium left. However, on the AGB thermohaline mixing can substantially increase the surface Li abundance above the Spite plateau value. It is therefore possible that low-mass AGB stars have contributed to the Galaxy's Li budget. The effect of a population of low-mass, low-metallicity lithium producers on Galactic chemical evolution models should be investigated.

These models do improve the agreement of the AGB models with observations of Li in carbon-enhanced metal-poor stars. We have extracted from the Stellar Abundances for Galactic Archaeology (SAGA) database (Suda et al. 2008) those CEMP stars that are both C-rich and have measured Li-abundances, and also that are still close to the main sequence turn-off (because first dredge-up will significantly reduce the surface $\mathrm{Li}$ abundance). We select only those stars in the metallicity range $-3<[\mathrm{Fe} / \mathrm{H}]<-2$ as the models presented herein may be expected to apply only over a limited range in metallicity. In particular, at very low metallicities, AGB stars can undergo additional mixing events not present in our models (see e.g. Fujimoto et al. 1990; Campbell \& Lattanzio 2008; Lau et al. 2009, among many others for a discussion of these mixing events). SAGA lists 5 turn-off objects with measured lithium abundances, the properties of which are displayed in Table 1.

One caveat should be added to the following discussion. The scenario of mass transfer from an AGB primary star on to a lower mass secondary in a binary system is expected to apply to those stars belonging to the CEMP-s subclass, i.e. those stars which have $[\mathrm{Ba} / \mathrm{Fe}]>1$ and $[\mathrm{Ba} / \mathrm{Eu}]>0.5$ according to the definitions given by Beers \& Christlieb (2005). The origin of the $r+s$ subclass of CEMP stars, which have $0<[\mathrm{Ba} / \mathrm{Eu}]<0.5$, is currently unknown. It is possible that their $s$-process enrichment has come from a binary mass transfer event, in which case the models presented herein would apply, but the enrichment may have another source entirely (see e.g. Lugaro et al. 2009, for a possible alternative formation scenario). This should be borne in mind throughout the following discussion.

We model CEMP stars by accreting material of the composition of the ejecta from our AGB models on to low-mass stars on the main sequence (see Stancliffe \& Glebbeek 2008, for details). We accrete $0.001,0.01$ and $0.1 \mathrm{M}_{\odot}$ of material on to a companion so that its final mass is $0.8 \mathrm{M}_{\odot}$, equivalent to the turn-off mass of the Halo. The model is then evolved until the star reaches the main-sequence turn-off, i.e. the point at which the Li-rich CEMP stars are observed. An example of accretion from the $1.5 \mathrm{M}_{\odot}$ model is shown in Fig. 2. 


\begin{tabular}{lccccr}
\hline Object & {$[\mathrm{Fe} / \mathrm{H}]$} & {$[\mathrm{C} / \mathrm{Fe}]$} & $\log _{10} \epsilon(\mathrm{Li})$ & $\log \mathrm{g}$ & Refs. \\
\hline CS 22964-161A & -2.41 & 1.35 & 2.09 & 3.7 & 1 \\
CS 22964-161B & -2.39 & 1.15 & 2.09 & 4.1 & 1 \\
HE 0024-2523 & -2.7 & 2.6 & 1.5 & 4.3 & 2 \\
CS 31080-095 & -2.85 & 2.69 & 1.73 & 4.5 & 3 \\
CS 31062-012 & -2.53 & 2.14 & 2.3 & 4.3 & 4 \\
\hline
\end{tabular}

Table 1. Properties of CEMP turn-off stars with measured Li-abundances, as extracted from the SAGA database. References: 1 - Thompson et al. (2008), 2 - Lucatello et al. (2003), 3 Sivarani et al. (2006), 4 - Aoki et al. (2008)

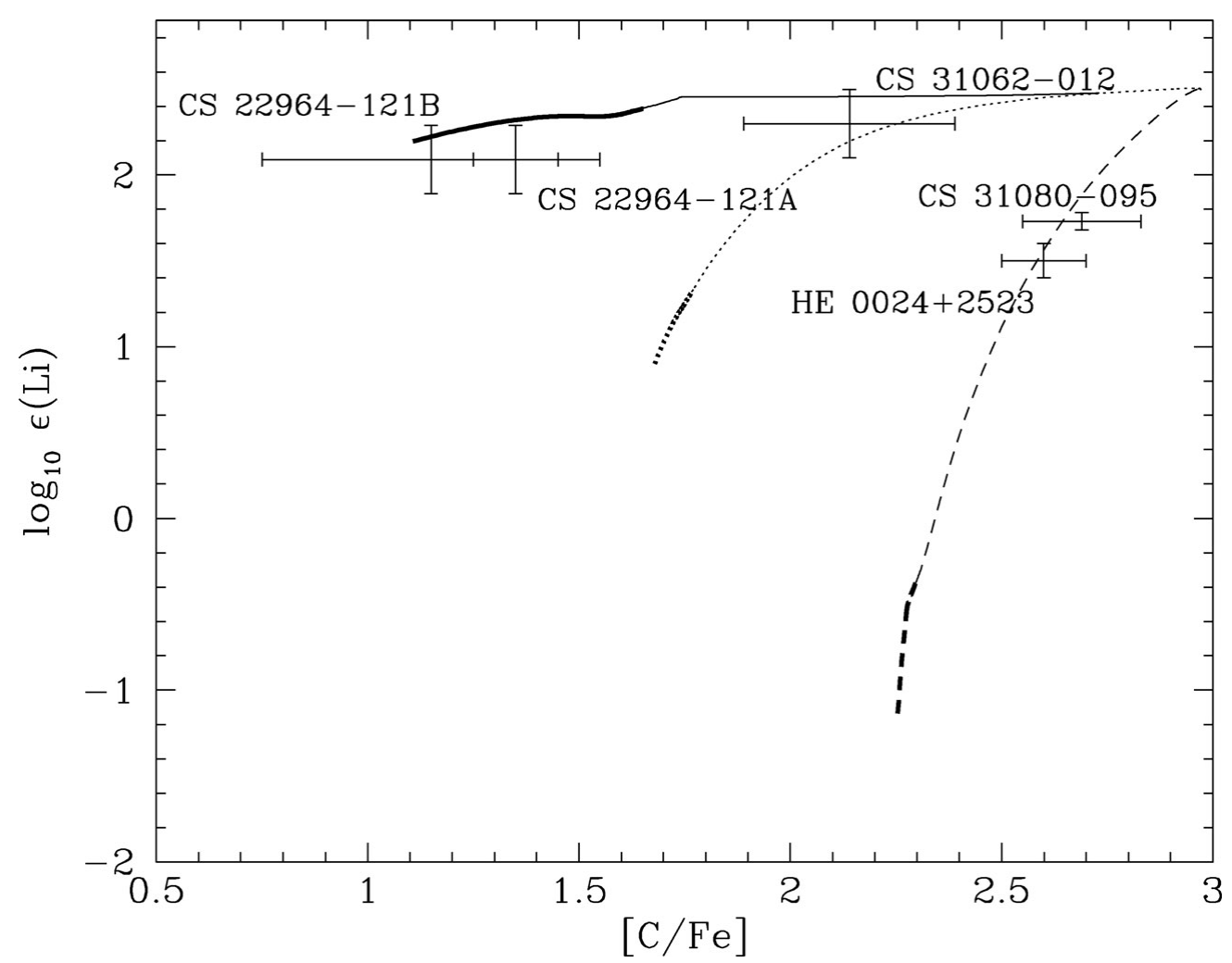

Figure 2. The evolution of $\log _{10} \epsilon(\mathrm{Li})$ with $[\mathrm{C} / \mathrm{Fe}]$ when accreting material from a $1.5 \mathrm{M}_{\odot}$ companion. The cases displayed are for when $0.001 \mathrm{M}_{\odot}$ (solid line), $0.01 \mathrm{M}_{\odot}$ (dotted line) and $0.1 \mathrm{M}_{\odot}$ (dashed line) is accreted. In each case, the secondary is left with a total mass of $0.8 \mathrm{M}_{\odot}$. Bold lines indicate where $\log g$ passes from 4.5 to 3.5 as the object evolves off the main sequence. The errorbars denote the locations of specific observed systems. The secondary is modelled including thermohaline mixing, gravitational settling and an extra turbulent process.

We find that a model that includes thermohaline mixing, gravitational settling and the ad hoc turbulent mixing of Richard et al. (2005) can reproduced the observed properties of CS 22964-121 if $0.001 \mathrm{M}_{\odot}$ of material is accreted, while for CS 31062-012 around $0.002 \mathrm{M}_{\odot}$ of material would have to be accreted. For CS 31080-095 and HE 0024+2523 a companion of between 1 and $1.5 \mathrm{M}_{\odot}$ mass is most likely required, though based on the high carbon abundance in these objects, it seems unlikely that the accreted material would have undergone any mixing. 


\section{Conclusions}

We have investigated the effect that thermohaline mixing has on the abundances of low-mass, low-metallicity AGB stars. We find that enough ${ }^{3} \mathrm{He}$ remains after the first giant branch that thermohaline mixing can still take place on the AGB. Thermohaline mixing can lead to substantial production of ${ }^{7} \mathrm{Li}$ - even up to abundances above the Spite plateau value. Thus it is possible to reconcile C- and Li-rich metal-poor stars with having come from a binary mass transfer scenario. The possibility that low-mass, lowmetallicity stars could be producers of lithium is intriguing and their role in Galactic chemical evolution should be investigated.

\section{Acknowledgements}

RJS is funded by the Australian Research Council's Discovery Projects scheme under grant DP0879472. This work was supported by the NCI National Facility at the ANU.

\section{References}

Abia, C. \& Isern, J. 1997, MNRAS, 289, L11

Anders, E. \& Grevesse, N. 1989, Geo.Cosmo.Acta, 53, 197

Aoki, W., Beers, T. C., Sivarani, T., Marsteller, B., Lee, Y. S., Honda, S., Norris, J. E., Ryan, S. G., \& Carollo, D. 2008, ApJ, 678, 1351

Beers, T. C. \& Christlieb, N. 2005, ARA\&A, 43, 531

Cameron, A. G. W. \& Fowler, W. A. 1971, ApJ, 164, 111

Campbell, S. W. \& Lattanzio, J. C. 2008, A\&A, 490, 769

Charbonnel, C. \& Zahn, J.-P. 2007, A\&A, 467, L15

Eggleton, P. P., Dearborn, D. S. P., \& Lattanzio, J. C. 2006, Science, 314, 1580

Eggleton, P. P., Dearborn, D. S. P., \& Lattanzio, J. C. 2008, ApJ, 677, 581

Fujimoto M. Y., Iben I. J., \& Hollowell, D. 1990, ApJ, 349, 580

Kippenhahn, R., Ruschenplatt, G., \& Thomas, H.-C. 1980, A\&A, 91, 175

Lau, H. H. B., Stancliffe, R. J. \& Tout, C. A. 2009, MNRAS, 396, 1046

Lebzelter, T., Lederer, M. T., Cristallo, S., Hinkle, K. H., Straniero, O., \& Aringer, B. 2008, A\&A, 486, 511

Lucatello, S., Gratton, R., Cohen, J. G., Beers, T. C., Christlieb, N., Carretta, E., \& Ramírez, S. 2003 , AJ, 125,875

Lugaro, M., Campbell, S. W., \& de Mink, S. E. 2009, PASA, 26, 322

Nollett, K. M., Busso, M., \& Wasserburg, G. J. 2003, ApJ, 582, 1036

Reimers, D. 1975, Memoires of the Societe Royale des Sciences de Liege, 8, 369

Richard, O., Michaud, G., \& Richer, J. 2005, ApJ, 619, 538

Sivarani, T., Beers, T. C., Bonifacio, P., Molaro, P., Cayrel, R., Herwig, F., Spite, M., Spite, F., Plez, B., Andersen, J., Barbuy, B., Depagne, E., Hill, V., François, P., Nordström, B., \& Primas, F. 2006, A\&A, 459, 125

Stancliffe, R. J. 2009, MNRAS, 394, 1051

Stancliffe, R. J., Church, R. P., Angelou, G. C., \& Lattanzio, J. C. 2009, MNRAS, 396, 2313

Stancliffe, R. J. \& Eldridge, J. J. 2009, MNRAS, 396, 1699

Stancliffe, R. J. \& Glebbeek, E. 2008, MNRAS, 389, 1828

Stancliffe, R. J., Glebbeek, E., Izzard, R. G., \& Pols, O. R. 2007, A\&A, 464, L57

Suda, T., Katsuta, Y., Yamada, S., Suwa, T., Ishizuka, C., Komiya, Y., Sorai, K., Aikawa, M., \& Fujimoto, M. Y. 2008, PASJ, 60, 1159

Thompson, I. B., Ivans, I. I., Bisterzo, S., Sneden, C., Gallino, R., Vauclair, S., Burley, G. S., Shectman, S. A., \& Preston, G. W. 2008, ApJ, 677, 556

Uttenthaler, S., Lebzelter, T., Palmerini, S., Busso, M., Aringer, B., \& Lederer, M. T. 2007, A\&A, 471, L41

Vassiliadis, E. \& Wood, P. R. 1993, ApJ, 413, 641 